\title{
IMPACT OF QUARANTINE, DUE TO COVID-19, ON PHYSICAL ACTIVITY, MONITORED WITH EEG (CASE STUDY)
}

\author{
Bastiurea Eugen ${ }^{1}$, Stan Zenovia ${ }^{2}$ \\ ${ }^{1,2}$ Human Performance Research Centre, Faculty of Physical Education and Sport,"Dunarea de \\ Jos" University of Galati, Romania \\ zenovia.stan@ugal.ro \\ eugen.bastiurea@ugal.ro
}

\begin{abstract}
Due to the situation created by the emergence of Covid-19, we found that the restrictions and quarantine had a significant effect on normal activities and especially on physical activities. Physical education teachers, coaches, sports instructors, etc., were forced to change the approach of this segment of activity, from all points of view. As the promotion of physical activity has become a global priority, leading to a drastic review of this field, we used the most advanced study methods to establish the effects of quarantine and motor activities at home on brain activity. Electroencephalogram (EEG) is a method that records electrical activity on the scalp and measures voltage changes resulting from ionic current flows in brain neurons. In this case study, we monitored the EEG activity of a Pilates instructor also the manifestation of brain waves (Delta, Theta, Alpha, Beta and Gamma) during specific activities during quarantine. We assumed that this forced adaptation to indoor activity will lead to certain manifestations of the brain waves depending on the specifics of the activities (theoretical training, physical training and online teaching Pilates sessions) and will be influenced by the extension of the quarantine period.
\end{abstract}

Keywords: Physical activities during COVID-19, EEG monitoring, online Pilates sessions

\section{Introduction}

In the current context, the new coronavirus disease (COVID-19) has a globally strong impact on all activities, and especially on physical activities. Thus, the WHO recommends $60 \mathrm{~min} /$ day of moderate physical activity for children 6 to 17 years old and $75 \mathrm{~min} /$ week of intense physical activity for adults. (Amri et al., 2020). The physical activity is an important factor with a beneficial effect on the health of the population, especially in the context of the arising anxiety associated with the new coronavirus: devastating effects of alcohol and drugs, extreme religious confrontations, depression and suicidal ideation, etc. (Sherman A. Lee, 2020). For this reason, we studied the way in which the specialists in motor activities faced these special requirements and how they managed to solve problems. We noticed that physical education teachers, coaches, sports instructors, etc., had major problems, especially emotional ones, due to the loss of physical contact with those they coordinated. The lack of physical body, touch, direct physical 
contact led to problems of lack of identity of the subject in question. (Valeria Varea \& Gustavo González-Calvo, 2020).

The effects of the pandemic also decisively influenced the activity of performance athletes as well as professionals in this field. (Schinke et al., 2020) For this reason, it is recommended that in the future, new ways be found to support these categories whose activity has a very important social impact (Papaioannou G. et al, 2020). The promotion of the physical activity has become a worldwide priority leading to a drastic review of physical activity and exercise during the COVID-19 pandemic (Chathuranga Ranasinghe et al, 2020). The results of recent studies have highlighted the close link between the effects of COVID-19 on people who have reduced physical activity and the weakening of the immune system. Thus, physical activity has become an important factor in reducing the risk of disease and immune system improvement (Barbara E. Ainsworth, Fuzhong Li, 2020). The most advanced study methods use the Electroencephalogram (EEG). This method represents the recording of electrical activity on the scalp and measures voltage changes resulting from ionic current flows in brain neurons (Noppadon Jatupaiboon et al., 2013).

It is already known that the physical exercise causes changes in brain's activity expressed by EEG models. The results of these studies show that due to these electrophysiological changes, all physiological, biochemical, cognitive, emotional, etc. mechanisms can be rearranged and restructured for the benefit of the person who practices physical activity (Moraes, 2007).

Because during this period, physical activity at home is recommended, with an emphasis on those simple exercises that can be performed in confined spaces and do not require complex equipment (Peijie Chen et al., 2020), in this case study we monitored the EEG of a Pilates instructor.

We aim to study the brain waves (Delta, Theta, Alpha, Beta and Gamma) during specific activities during quarantine. We assumed that this forced adaptation will lead to certain manifestations of the brain waves depending on the specifics of the activities (theoretical training, physical training and online teaching Pilates sessions) and will be influenced by the extension of the quarantine period.

\section{Methods}

Between April and June 2020, we monitored the EEG activity of a Pilates instructor obliged to carry out his activity in quarantine, on three activities performed at home: indoors physical activity (PACS), online physical activity - Pilates (OPAP) and theoretical training activity 
(TTA).We monitored 12 sessions from each activity by selecting those with an approximately equal duration (60 minutes).

We used MindWave Mobile 2 device (fig. 1) with recording on an eSense counter in the Meditation Journal - Microsoft Silverlight (fig. 2). The domains pursued by the EEG were the specific eSense (attention and relaxation) each with values in all frequencies of brain waves. For each different type of eSense, we reported the value of the meter on a relative scale from 1 to 100 (fig. 3).

MindWave Mobile 2 securely measures and transmits EEG power spectra (Alpha waves, Beta waves, etc.), NeuroSky eSense meters (attention and relaxation) and eye blinking. The device consists of a headset, an ear clip and a sensor arm. The reference and grounding electrodes of the headphones are on the ear clip, and the EEG electrode is on the sensor arm, placed on the forehead above the eye.

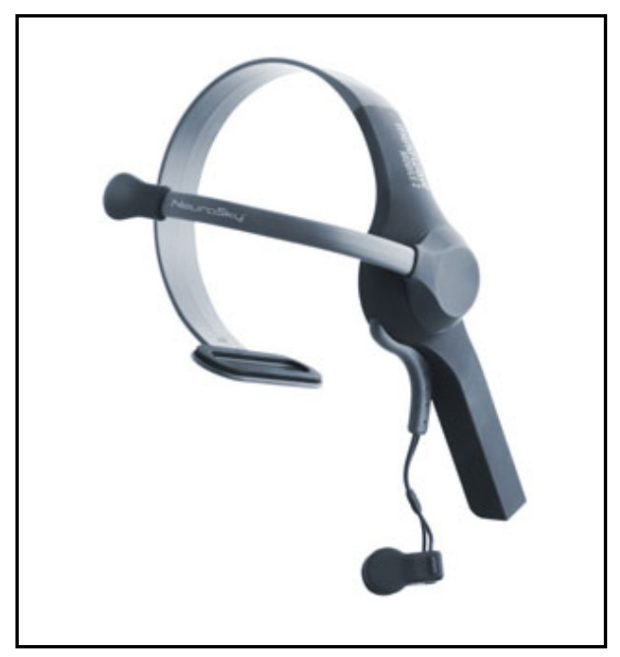

Figure 1. MindWave Mobile 2

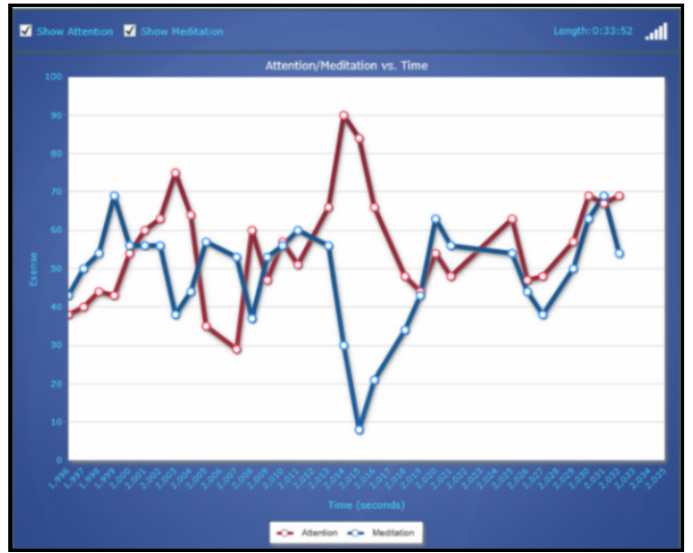

Figure 2. Counter NeuroSky eSense

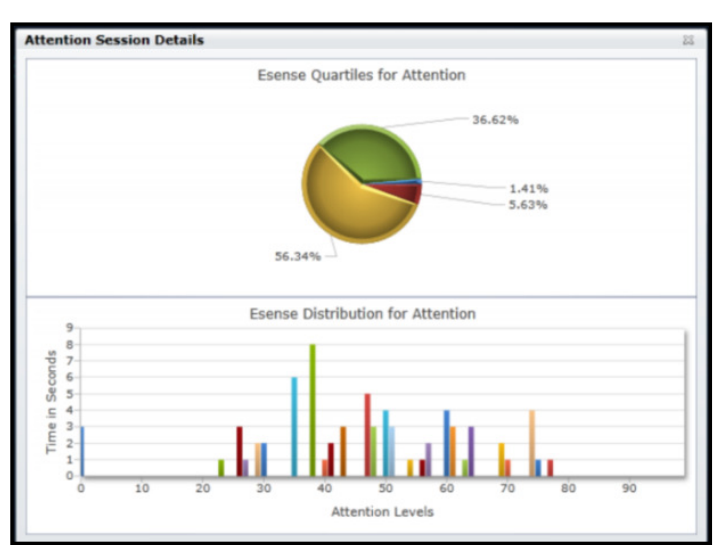

Figure 3. Attention Session Details 
The electroencephalogram (EEG) is a recording of the electrical activity of the brain in the scalp. The recorded waveforms reflect cortical electrical activity which is quite small and has certain frequencies: Delta, has a frequency of $3 \mathrm{~Hz}$; Theta, has a frequency between $3.5-7.5 \mathrm{~Hz}$ and is classified as "slow" activity; Alpha, has a frequency between 7.5 -13 Hz.; Beta is a "fast" activity; it has a frequency between 14-30Hz.; Low range: with frequencies between 30-200 Hz.

\section{Results and Discussions}

The data we extracted from the eSense graphical representation (fig. 3) was entered in the tables, separately for attention and relaxation, depending on each monitored activity.

In the Table 1 we showed the EEG values during indoors physical activity (PACS). We noticed in the Graphs 1 and 2, small percentages at frequencies of $0-25 \mathrm{~Hz}$ and $75-100 \mathrm{~Hz}$. They are the extremes of the mental work field: low frequencies (Delta, Theta, Alpha and Beta low - 0-25Hz) and high frequency oscillations (high Gamma with values over $60 \mathrm{~Hz}$ showing ultrafast EEG activity). EEG frequencies are mostly in the $25-75 \mathrm{~Hz}$ range. The efficiency is between 70 and $88.3 \%$ of the measured time.

Table 1. EEG values recorded during indoors physical activity (PACS)

\begin{tabular}{|c|c|c|c|c|c|c|c|c|c|c|c|c|}
\hline \multicolumn{13}{|c|}{ PHYSICAL ACTIVITY IN A CLOSED SPACE (PACS) } \\
\hline \multicolumn{13}{|c|}{ Attention \% } \\
\hline Hz Nr. & 1 & 2 & 3 & 4 & 5 & 6 & 7 & 8 & 9 & 10 & 11 & 12 \\
\hline O-25Hz & 7,88 & 7,47 & 4,49 & 3,73 & 7,8 & 2,3 & 9,81 & 10,54 & 3,77 & 2,63 & 3,1 & 6,36 \\
\hline $25-50 H z$ & 45,32 & 50,23 & 42,88 & 38,7 & 53,5 & 16,22 & 41,78 & 53,67 & 38,27 & 53,53 & 52,01 & 44,86 \\
\hline $50-75 H z$ & 39,42 & 37,14 & 46,15 & 50,63 & 35,43 & 61,47 & 42,18 & 33,69 & 49,45 & 40,26 & 29,88 & 40,99 \\
\hline $75-100 H z$ & 7,38 & 5,16 & 6,47 & 6,93 & 3,26 & 20,28 & 6,23 & 2,1 & 8,51 & 3,59 & 3,1 & 7 \\
\hline \multicolumn{13}{|c|}{ Relaxation \% } \\
\hline $\mathbf{H z} N \mathrm{Nr}$. & 1 & 2 & 3 & 4 & 5 & 6 & 7 & 8 & 9 & 10 & 11 & 12 \\
\hline o-25Hz & 7,05 & 2,98 & 2,58 & 9,72 & 4,26 & 6,08 & 4,08 & 5,87 & 0,89 & 2,18 & 8,82 & 5,03 \\
\hline $25-50 H z$ & 48,19 & 36,34 & 34,24 & 57,57 & 41,8 & 36,82 & 37,99 & 39,55 & 27,01 & 35,71 & 43,96 & 46,79 \\
\hline $50-75 H z$ & 40,74 & 53,87 & 53,82 & 30,6 & 47,97 & 51,48 & 50,6 & 49,31 & 61,77 & 53,27 & 44,12 & 42,29 \\
\hline $75-100 \mathrm{~Hz}$ & 6,02 & 6,81 & 9,36 & 2,1 & 5,97 & 5,62 & 7,33 & 5,27 & 10,33 & 8,85 & 3,1 & 5,89 \\
\hline Efic. \% & 80,00 & 84,70 & 88,30 & 83,30 & 70,00 & 78,50 & 86,20 & 84,10 & 83,30 & 74,20 & 72,00 & 83,41 \\
\hline
\end{tabular}
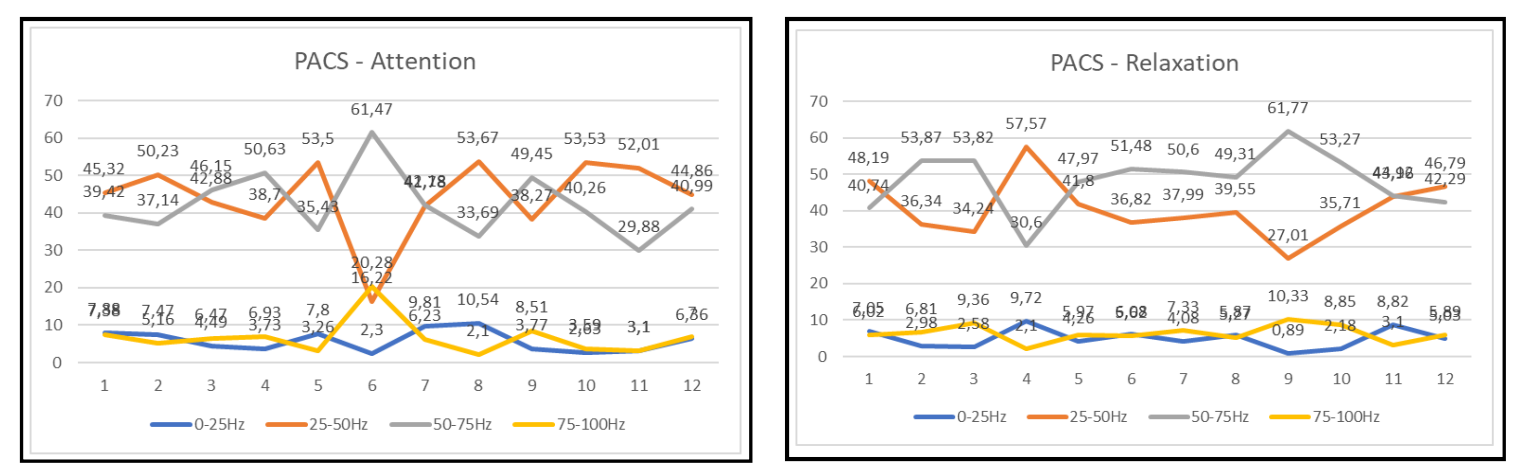

Graph 1. Evolution of EEG for attention (PACS)

Graph 2. Evolution of EEG for relaxation (PACS)

In session 6 (chart 1) we noticed a clear decrease in frequencies of 0-5 Hz and a significant increase in those of $50-100 \mathrm{~Hz}$. In this session we elaborated a new structure of a Pilates session (more elaborate and structured exercises on another framework) requiring fast EEG activity - the 
highest percentage of extreme frequencies $-22.28 \%$. At the basic frequencies $25-75 \mathrm{~Hz}$, we noticed that they alternated depending on the specifics of the physical training. While in session 4 and 9 for attention, the frequencies do not fluctuate, for relaxation we noticed a peak inside the range of $50-75 \mathrm{~Hz}$ and a decrease for the range of $25-50 \mathrm{~Hz}$. In Table 2 we showed the EEG values recorded for the physical activity during online Pilates sessions (OPAP).

Table 2. EEG values recorded during physical activity during online Pilates sessions

\begin{tabular}{|c|c|c|c|c|c|c|c|c|c|c|c|c|}
\hline \multicolumn{13}{|c|}{ ONLINE PHYSICAL ACTIVITY - Pilates (OPAP) } \\
\hline \multicolumn{13}{|c|}{ Attention $\%$} \\
\hline $\mathbf{H z} \sim \mathbf{N r}$. & 1 & 2 & 3 & 4 & 5 & 6 & 7 & 8 & 9 & 10 & 11 & 12 \\
\hline $0-25 H z$ & 3,98 & 14,5 & 4,53 & 9,77 & 3,66 & 6,38 & 4,37 & 5,83 & 7,01 & 7,68 & 4,87 & 5,39 \\
\hline $25-50 H z$ & 43,03 & 54,94 & 52,76 & 55,19 & 50,73 & 43,28 & 47,64 & 45,88 & 53,82 & 46,24 & 44,95 & 50,15 \\
\hline $50-75 H z$ & 47,84 & 27,88 & 35,88 & 32,13 & 36,64 & 42,55 & 44,44 & 43,22 & 36,31 & 39,94 & 46,44 & 41,3 \\
\hline $75-100 H z$ & 5,15 & 2,69 & 4,53 & 2,91 & 3,66 & 7,78 & 3,55 & 5,07 & 2,87 & 6,14 & 3,74 & 3,16 \\
\hline \multicolumn{13}{|c|}{ Relaxation \% } \\
\hline $\mathrm{Hz} \sim \mathrm{Nr}$ & 1 & 2 & 3 & 4 & 5 & 6 & 7 & 8 & 9 & 10 & 11 & 12 \\
\hline $0-25 H z$ & 1,94 & 4,92 & 4,46 & 2,06 & 3,38 & 3,52 & 1,51 & 3,41 & 0,96 & 10,2 & 3,62 & 4,69 \\
\hline $25-50 H z$ & 33,37 & 38,37 & 42,35 & 33,97 & 35,66 & 45,82 & 28,31 & 47,54 & 27,83 & 55,56 & 39,05 & 32 \\
\hline $50-75 \mathrm{~Hz}$ & 57,22 & 49,98 & 47,9 & 55,12 & 52,81 & 47,63 & 58,92 & 44,85 & 59,52 & 33,08 & 49,81 & 2 \\
\hline $75-100 H z$ & 7,47 & 6,72 & 5,29 & 8,86 & 8,15 & 3,02 & 11,26 & 4,2 & 11,69 & 1,16 & 7,52 & 2,96 \\
\hline Efic. \% & 85,90 & 84,68 & 50,45 & 52,70 & 52,70 & 52,50 & 56,40 & 85,00 & 83,00 & 84,00 & 86,00 & 84,88 \\
\hline
\end{tabular}

We noticed in the Graphs 3 and 4 small percentages for frequencies between $0-25 \mathrm{~Hz}$ and $75-$ $100 \mathrm{~Hz}$. There are extremes of the mental field of work: low frequencies (Delta, Theta, Alpha and Beta low - 0-25Hz) and high frequency oscillations (high Gamma with values over $60 \mathrm{~Hz}$ that show ultrafast EEG activity, in this case with a higher percentage for relaxation). The activity is pleasantly and safely performed. EEG frequencies are mostly in the $25-75 \mathrm{~Hz}$ range.

The efficiency is between $50.45 \%$ and $86 \%$ of the measured time. We noticed that after 2 sessions the efficiency decreases drastically and then increases progressively towards the end of the period. Starting with session 3 there were several people so it was necessary to make certain adaptations to the activity. We noticed a predominance on $25-50 \mathrm{~Hz}$ and $0-25 \mathrm{~Hz}$ at the beginning of the period and approximately equal values between the low frequencies $0-25 \mathrm{~Hz}$ and the maximum ones of $75-100 \mathrm{~Hz}$. For the relaxation component we noticed a compensation of the activity on attention and a predominance at the frequencies $75-100 \mathrm{~Hz}$.
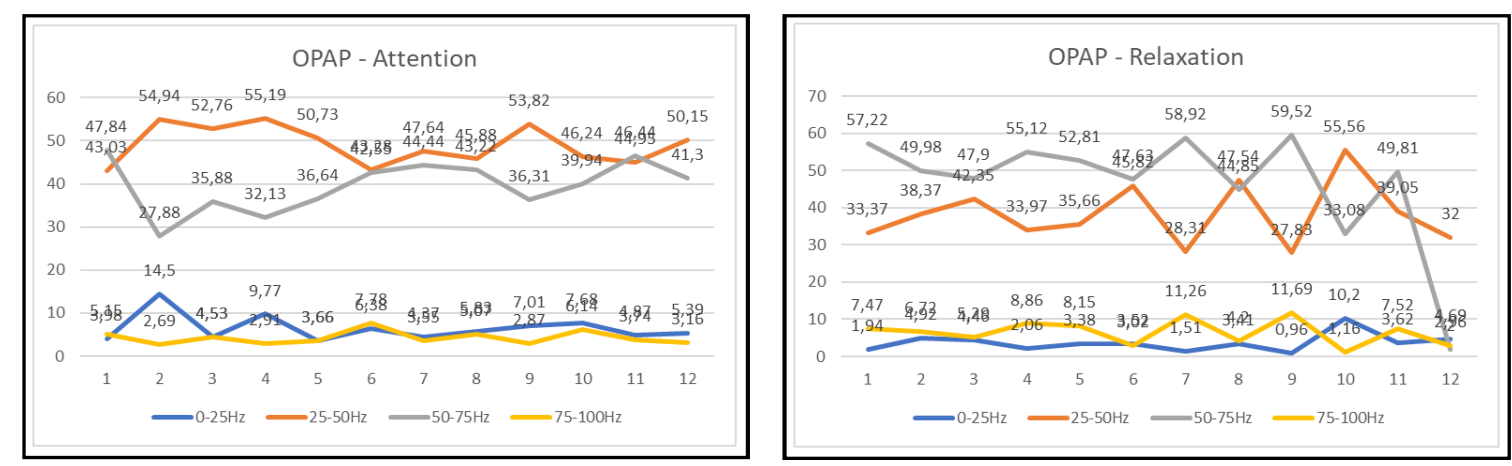

Graph 3. Evolution of EEG for attention (OPAP)

Graph 4. Graph 4. Evolution of EEG for relaxation (OPAP) 
In the Table 3 we showed the EEG values recorded during the theoretical training activities (TTA).

Table 3. EEG values recorded during theoretical training activities

\begin{tabular}{|l|l|l|l|l|l|l|l|l|l|l|l|l|}
\hline \multicolumn{10}{|c|}{ THEORETICAL TRAINING ACTIVITY (TTA) } \\
\hline \multicolumn{10}{|c|}{$\mathbf{1 0}$} \\
\hline $\mathbf{1}$ & $\mathbf{2}$ & $\mathbf{3}$ & $\mathbf{4}$ & $\mathbf{5}$ & $\mathbf{6}$ & $\mathbf{7}$ & $\mathbf{8}$ & $\mathbf{9}$ & $\mathbf{1 0}$ & $\mathbf{1 1}$ & $\mathbf{1 2}$ \\
\hline $\mathbf{0 - 2 5 H z}$ & 4,28 & 8,8 & 8,27 & 10,09 & 9,17 & 11,53 & 5,74 & 11,01 & 12,58 & 7,23 & 14,36 & 9,04 \\
\hline $\mathbf{2 5}-\mathbf{5 0 H z}$ & 34,5 & 28,52 & 40,55 & 49,25 & 39,96 & 49,91 & 39,99 & 52 & 49,37 & 46,88 & 47,08 & 42,99 \\
\hline $\mathbf{5 0 - 7 5 H z}$ & 51,24 & 46,46 & 43,06 & 34,52 & 42,62 & 34,23 & 46,23 & 34,59 & 33,55 & 40,96 & 33,84 & 39,7 \\
\hline $\mathbf{7 5 - 1 0 0 H z}$ & 9,98 & 16,23 & 8,12 & 6,14 & 9,17 & 4,32 & 8,04 & 2,4 & 4,5 & 4,93 & 4,71 & 8,27 \\
\hline
\end{tabular}
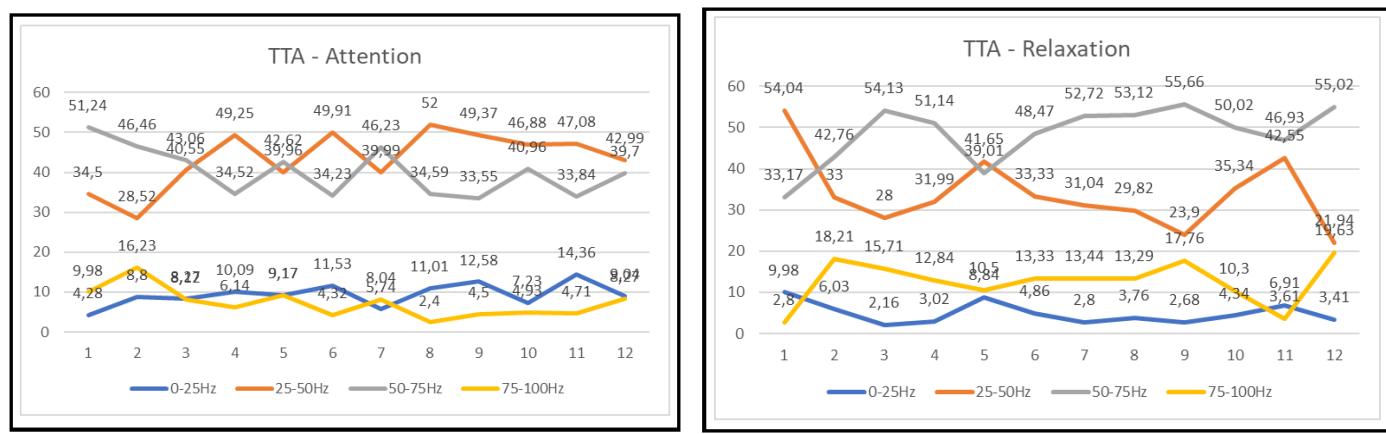

Graph 5. Evolution of EEG for attention (TTA)

Graph 6. Evolution of EEG for relaxation (TTA)

We noticed high values at the efficiency of $77.08 \%-86.35 \%$. the lowest value is also found at the session 6. In the Graphs 5 and 6 we noticed a predominance of frequencies $-25-50 \mathrm{~Hz}$ and 0 $25 \mathrm{~Hz}$ and a compensation of the predominance of the attention frequencies $-50-75 \mathrm{~Hz}$ and $75-$ $100 \mathrm{~Hz}$. In the Graph 7 we showed the efficiency values for all three activities. We noticed the highest efficiency at TTA and the lowest at online teaching (OPAP), and a small value at the first session with more subjects and then, a slow progressive growth. After session 6 in which the sessions were changed, we noticed a jump to values of $80 \%$. The values for PACS and TTA were fairly linear. 


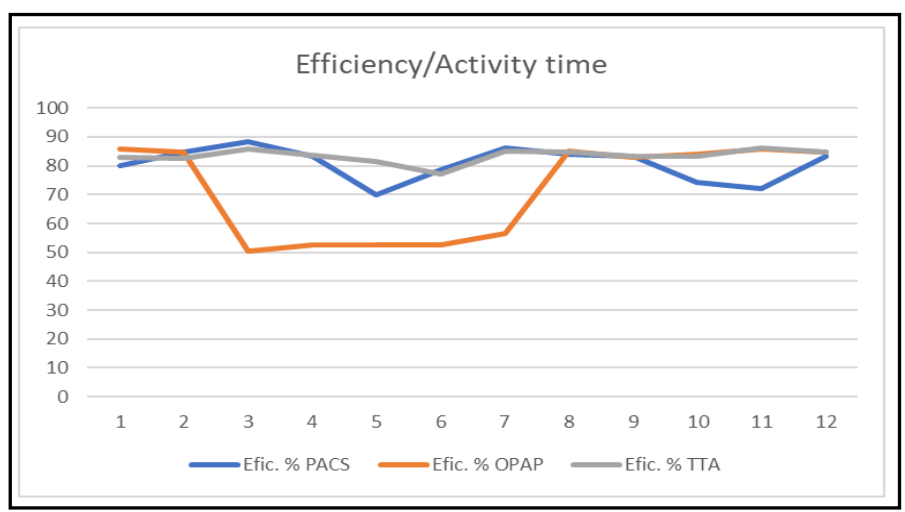

Graph 7. EEG evolution for efficiency

In the Graphs 8, 9, 10 and 11 we showed the values of the frequency categories $(0-100 \mathrm{~Hz})$ on both data categories (attention and relaxation). In the Graph $8(0-25 \mathrm{~Hz})$ we showed the most significant line at TTA for attention and the lowest at OPAP for relaxation. In the Graph 9 (25$50 \mathrm{~Hz}$ ) we showed the most significant line at OPAP for attention and the lowest at TTA for relaxation.

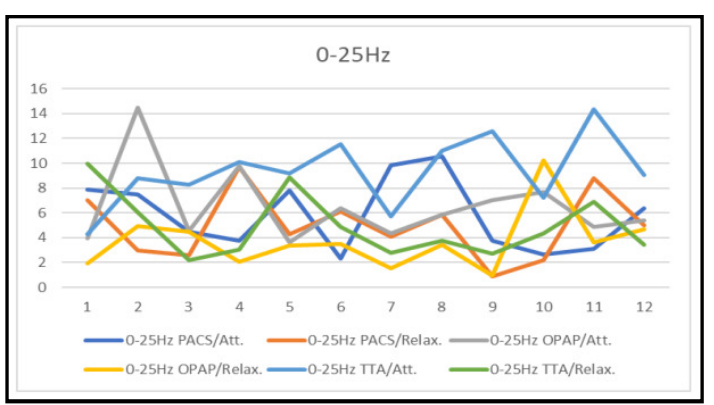

Graph 8. EEG evolution for $0-25 \mathrm{~Hz}$ frequencies

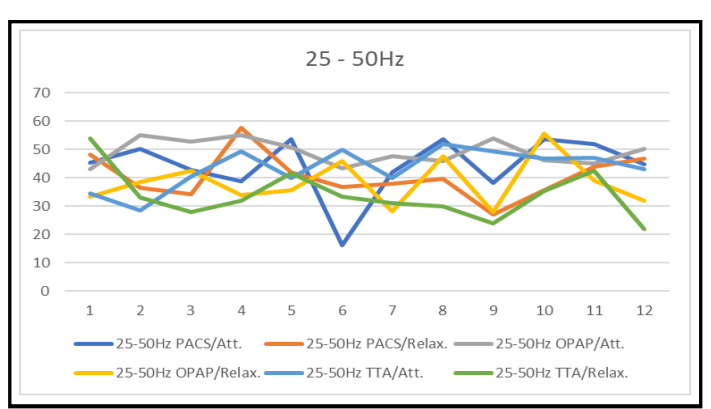

Graph 9. EEG evolution for $25-50 \mathrm{~Hz}$ frequencies

In the Graph $10(50-75 \mathrm{~Hz})$ we showed the most significant line at PACS for relaxation and the lowest at OPAP for attention. In the Graph $11(75-100 \mathrm{~Hz})$ we showed the most significant line at TTA for relaxation and the lowest at OPAP for attention.

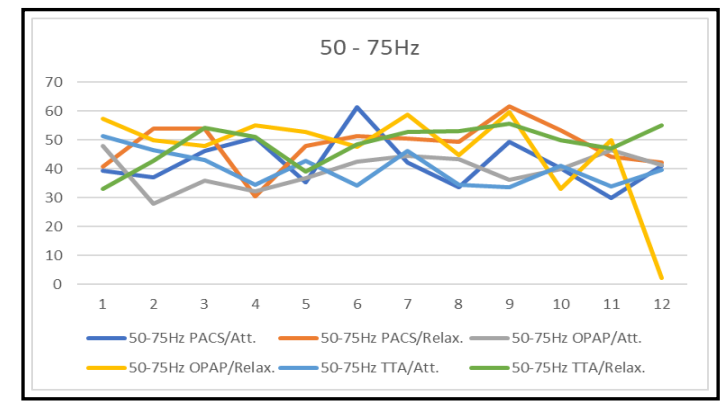

Graph 10. EEG evolution for 50-75Hz frequencies

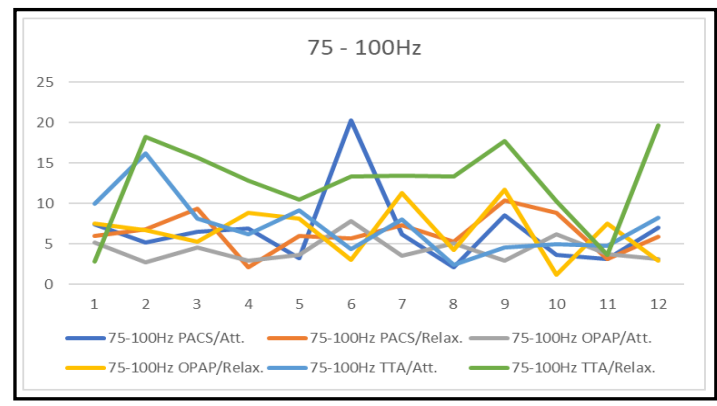

Graph 11. EEG evolution for $75-100 \mathrm{~Hz}$ frequencies 
We noticed in people who perform physical activity, a strong correlation between participants' perception of exercise intensity and fatigue due to hyperthermia (Nielsen B, Nybo L., 2003). The effects are much more visible when physical activity takes place indoors. Strictly referring to physical activity and EEG, we know that Delta waves in excess can cause brain damage, learning problems and inability to think while an optimal level improves the immune system and ensures deep sleep. Excess Theta waves induce hyperactivity, impulsivity and lack of attention and if they are at a low level, anxiety and stress appear. If Theta waves are at an optimal level, creativity, intuition and relaxation are induced.

Alpha waves in excess induce an inability to concentrate and too low level give a state of anxiety and strong stress. In the case of Beta waves, high level induces high excitement and inability to relax and too low level induces depression.

If the level is optimal, there is a conscious focus and a good memory. The waves with the highest frequency - Gamma will induce stress and anxiety if their level is high. If the level is optimal, cognition, learning, perception and information processing occur. Low values for Gamma showed a depressed state or even ADHD (Preeti Gupta Vishal Aditya, 2020). According to other studies, Theta waves ensure the storage of new memories and the coordination of memory (Wolfgang Klimesch, 1990). High Gamma waves(50-70Hz) are associated with cognitive tasks (hearing, reading and speaking) and when it rises to $100 \mathrm{~Hz}$ it is suitable for emotions (M. Li and B. Lu, 2009).

\section{Conclusions}

Based on the data recorded after EEG monitoring, we confirmed our research hypothesis. Brain waves had different values on the two characteristics (attention and relaxation) in all three activities performed at home during the quarantine period due to COVID-19. The monitored subject was relaxed in the case of theoretical training (TTA) and had a good level of Theta waves (creativity and intuition). For the physical training sessions (PACS), in session 6 we clearly observed a decrease of the frequencies of $0-25 \mathrm{~Hz}$ and the significant increase of those of 50$100 \mathrm{~Hz}$. In this session, we elaborated a new structure of a Pilates session (more elaborate and structured exercises on another framework) requiring fast EEG activity - the highest percentage of frequencies at extremes $-22.28 \%$.

The Pilates sessions conducted online, due to the fact that they represented an absolutely new activity, showed an intense brain activity of the subject and was demonstrated by an ultra-fast EEG activity. We noticed the highest efficiency is at TTA and the lowest at online teaching (OPAP). We noticed a small value, a lack of distributive attention at the first session with several 
subjects and then, a slow progressive growth. After the sixth session, in which the subject changed the structure of the sessions and adapted the activity as necessary, there was a jump to $80 \%$ for the efficiency. The subject quickly adapted to the special requirements due to the fact that he changed his working conditions, adapted his physical effort and progressed over time.

\section{Bibliography}

1. Amir Jalaly Bidgoly, Hamed Jalaly Bidgoly, Zeynab Arezoumand. 2020. A survey on methods and challenges in EEG based authentication. Computers \& Security, Volume 93, 101788, ISSN 0167-4048.

2. Amri Hammami, Basma Harrabi, Magni Mohr \& Peter Krustrup. 2020. Physical activity and coronavirus disease 2019 (COVID-19): specific recommendations for home-based physical training. Managing Sport and Leisure.

3. Barbara E. Ainsworth, Fuzhong Li. 2020. Physical activity during the coronavirus disease-2019 global pandemic. Journal of Sport and Health Science, Volume 9, Issue 4, Pages 291-292, ISSN 2095-2546.

4. Chathuranga Ranasinghe, Cemal Ozemek \& Ross Arena. 2020. Exercise and well-being during COVID 19 - time to boost your immunity. Expert Review of Anti-infective Therapy.

5. M. Li and B. Lu. 2009. Emotion classification based on gamma-band EEG. Annual International Conference of the IEEE Engineering in Medicine and Biology Society, Minneapolis, MN, 2009, pp. 1223-1226.

6. Moraes Helena, Ferreira Camila, Deslandes Andréa, Cagy Mauricio, Pompeu Fernando, Ribeiro Pedro, \& Piedade Roberto. 2007. Beta and alpha electroencephalographic activity changes after acute exercise. Arquivos de Neuro-Psiquiatria, 65(3a), 637-641.

7. Nayak CS, Anilkumar AC. 2020. EEG Normal Waveforms. In: StatPearls [Internet]. Treasure Island (FL): StatPearls Publishing.

8. Nielsen B, Nybo L. 2003. Cerebral changes during exercise in the heat. Sports Med. 33(1):1-11. PMID: 12477374.

9. Noppadon Jatupaiboon, Setha Pan-ngum, Pasin Israsena. 2013. Real-Time EEG-Based Happiness Detection System. The Scientific World Journal, Article ID 618649, 12 pages.

10. Papaioannou, R. J. Schinke, Y. K. Chang, Y. H. Kim \& J. L. Duda. 2020. Physical activity, health and well-being in an imposed social distanced world. International Journal of Sport and Exercise Psychology, 18:4, 414-419.

11. Peijie Chen, Lijuan Mao, George P. Nassis, Peter Harmer, Barbara E. Ainsworth, Fuzhong Li. 2020. Coronavirus disease (COVID-19): The need to maintain regular physical activity while taking precautions. Journal of Sport and Health Science, Volume 9, Issue 2, ISSN 2095-2546.

12. Preeti Gupta Vishal Aditya. 2020. Brain Computer Interface \& its Applications. (ASET) B.Tech (CSE, VIII Semester) AMITY SCHOOL OF ENGINEERING \& TECHNOLOGY AMITY UNIVERSITY RAJASTHAN.

13. Sherman A. Lee. 2020. Coronavirus Anxiety Scale: A brief mental health screener for COVID-19 related anxiety. Death Studies, 44:7, 393-401.

14. Wolfgang Klimesch. 1999. EEG alpha and theta oscillations reflect cognitive and memory performance: a review and analysis. Brain Research Reviews, Volume 29, Issues 2-3, 1999, Pages 169-195. 